\title{
Compositos de Bioplástico y Fibras Celulósicas como Reemplazo de Tableros Fabricados con Resinas Sintéticas
}

\section{Composites of Bioplastic and Cellulosic Fibers as a Replacement for Boards Made with Synthetic Resins}

\section{Doctorando:}

\author{
Ing. Natalia Edith Suárez \\ Mención: Materiales (UTN-FRLP) \\ Instituto de Investigaciones en Catálisis y Petroquímica (INCAPE) \\ suareznataliae@gmail.com / nsuarez@fiq.unl.edu.ar
}

\section{Director:}

\section{Dr. Alberto Julio Marchi}

Instituto de Investigaciones en Catálisis y Petroquímica (INCAPE)

\section{Co-Directora:}

\section{Dra. Laura Battaglia}

Centro de Investigaciones de Métodos Computacionales (CIMEC)

\section{Resumen}

El objetivo general de esta tesis de doctorado es desarrollar materiales compuestos WBPC (Wood Bio-Plastic Composite), a partir de la mezcla de uno o más biopolímeros (matriz), fibras celulósicas (refuerzo) y aditivos o mejoradores químicos (agentes entrecruzantes, ignífugos, protectores de radiación UV, pigmentos, agentes antimicrobianos, etc.). Al presente, se han obtenido biocompositos con una matriz de proteína de soja entrecruzada con glutaraldehído y reforzada con aserrín de madera de eucalipto, empleando moldeo por termocompresión. Los mismos presentan propiedades físicas y mecánicas que cumplen con especificaciones de productos comerciales de similares características y un buen acabado superficial. El agregado de aserrín y de agente entrecruzante a la matriz bioplástica aumenta la rigidez, la dureza y el módulo de ruptura de estos WBPCs, así como su resistencia a la degradación por la acción del medio ambiente. Además, luego de 24 h de inmersión en agua, los WBPCs preparados pueden recuperar prácticamente sus dimensiones originales luego del secado en estufa a $105{ }^{\circ} \mathrm{C}$. Como trabajo futuro se planea obtener probetas de WBPCs con una matriz mixta de bioplásticos, a partir de proteína de soja y quitosano y, además, modelar con herramientas de simulación numérica el comportamiento en flexión de estos materiales.

Palabras claves: biocomposito, bioplástico, proteína de soja, fibras celulósicas, termocompresión.

\begin{abstract}
The general objective of this doctoral thesis is to develop WBPC (Wood Bio-Plastic Composite) composite materials, from the mixture of one or more biopolymers (matrix), cellulosic fibers (reinforcement) and chemical additives (crosslinking agents, flame retardants, UV radiation protectors, pigments, antimicrobialagents, etc.). At the time being, WBPCs made of soy protein crosslinked with glutaraldehyde and reinforced with sawdust have been obtained by thermocompression. These biocomposites have physical and mechanical properties that meet commercial product specifications and also good surface finish. The addition of sawdust and crosslinking agent to the bioplastic matrix increases the stiffness, hardness and modulus of rupture of these WBPCs, as well as their resistance to degradation by the action of the environment. In addition, after 24 hours of immersion in water; the
\end{abstract}


prepared WBPCs can practically recover their original dimensions by drying them in an oven at $105^{\circ} \mathrm{C}$. As future work, it is planned to obtain WBPC specimens with a mixed matrix of bioplastics, specifically, soybean protein and chitosan, and, to model the flexural behavior of these materials with numerical simulation tools.

Keywords: biocomposite, bioplastic, soy protein, cellulosic fibers, thermocompression.

\section{Introducción}

En la actualidad, se puede encontrar una gran variedad de materiales compuestos producidos a partir de diversas matrices poliméricas, generalmente derivadas del petróleo, a las que se les agrega un material de refuerzo como, por ejemplo, fibras de vidrio, de carbón u otros materiales sintéticos. El elevado costo de producción de estos materiales, provenientes de fuentes no renovables, hace necesario encontrar materias primas alternativas. Precisamente, en otros tipos de materiales compuestos más amigables con el medio ambiente (Netravali y Chabba, 2003), también denominados biocompositos (Mitra, 2014), se utilizan fibras obtenidas a partir de recursos renovables como, por ejemplo, la madera. El empleo de ésta como material de refuerzo reduce el costo total de los materiales compuestos en comparación a los basados en fibras sintéticas.

La madera, generalmente en forma de aserrín o finos, se emplea en combinación con polímeros termoplásticos como, por ejemplo, PE, PP, PET, PVC (virgen o reciclado) bajo condiciones controladas de calor y presión. De esta manera, se obtienen los denominados WPCs (Wood Plastic Composites), a los cuales es necesario añadir aditivos para mejorar la adhesión entre este tipo de fibras hidrofílicas y la matriz hidrófoba (Schwarzkopf y Burnard, 2016; Rahman et al., 2013; Selke y Wichman, 2004). Los WPCs son una mezcla homogénea de material sintético y de material biobasado en íntimo contacto, por lo que son difíciles de reciclar. La encapsulación de la madera por parte de la matriz de polímero no biodegradable conduce a que estos materiales compuestos, en su conjunto, tampoco lo sean (Chan et al., 2017).

La madera también se emplea en combinación con polímeros termoestables para obtener compositos como la madera terciada o contrachapado (PlywoodTM), los tableros de partículas o aglomerados ("particleboard"), los tableros de virutas orientadas (OSB), el cartón de madera ("hard-board") y el MDF ("médium-density fiberboard") (Stark et al., 2010). En estos casos, se utilizan resinas de fenol-formaldehído o urea-formaldehído como adhesivos o agentes de cohesión y para aumentar la resistencia a la humedad. A este respecto, es importante tener en cuenta que hoy en día se recomienda evitar el uso de formaldehído, por considerarlo un compuesto de alta toxicidad y con propiedades cancerígenas (American Cancer Society, 2014). Además, el origen de las resinas mencionadas anteriormente sigue siendo de derivados del petróleo. Esto implica no solo el uso de un recurso natural no renovable, sino que también se contribuye a la profundización de la contaminación ambiental generada durante la disposición final de estos plásticos debido a su muy baja biodegradabilidad. Los usos más comunes de estos materiales compuestos son en revestimientos, paneles divisorios y amoblamientos.

Una variante son los ecocompositos formados por biopolímeros y fibras naturales. Estos presentan ventajas medioambientales y ecológicas respecto de los compositos convencionales, ya que tanto la matriz como el refuerzo se obtienen a partir de recursos renovables y son biodegradables, lo que facilita su disposición al finalizar su vida útil (Mitra, 2014; Bogoeva-Gaceva et al., 2007). Existe una gran variedad de matrices biodegradables, tanto naturales como sintéticas, entre las que se pueden mencionar PLA (ácido poliláctico), PHA (polihidroxialcanoatos), almidón termoplástico, etc. La mayoría de estos polímeros se degrada a través de reacciones enzimáticas cuando se someten a un ambiente de compost, y otros por ataque bacterial o microbiano en ambientes húmedos (Netravali and Chabba, 2003). Al respecto, resultan de particular interés los ecocompositos basados en fibra de madera, también conocidos como WBPCs (Wood Bio-Plastic Composites).

Un factor que hace aún más atractivo el empleo de estos biocompositos es que se pueden obtener a partir de residuos o subproductos de bajo valor comercial de algunas actividades económicas o industrias regionales. En este sentido, se está estudiando la producción de WBPCs a partir de aserrín y excedentes de harina de soja, provenientes de las industrias maderera y aceitera, respectivamente, de la región del litoral. Un material alternativo es el quitosano, el cual se obtiene del exoesqueleto de los crustáceos marinos cuyos descartes representan un residuo de la industria pesquera en la zona costera.

La harina de soja es un subproducto de la extracción del aceite de soja, de la cual se puede obtener aislado de proteína de soja (SPI, soy protein isolate). De acuerdo con Song et al. (2011) el SPI presenta una buena biodegradabilidad, biocompatibilidad y procesabilidad por lo que resulta un buen candidato para reemplazar a los polímeros sintéticos. Sin embargo, sus propiedades mecánicas y su resistencia al agua deben ser mejoradas: el entrecruzamiento es una de las formas propuestas para lograr estas mejoras. Entre los entrecruzantes más utilizados se hallan los dialdehídos (Bai and Liu X, 2017; Wong and Jameson, 2012). En particular, el glutaraldehído (GTA) es empleado como entrecruzante de SPI ya sea para formar adhesivos mejorados (Wang et al. 2006) o 
packaging biodegradable (Park et al. 2000). También es ampliamente conocido para entrecruzar quitosano [Li et al, 2013; Kildeeva et al, 2009], por lo que la propuesta de una matriz mixta de SPI y quitosano resulta atractiva ya que, ambos cuentan con grupos amino en su estructura química, los cuales se pueden entrecruzar con el mismo agente reticulante.

Tanto un mercado de materiales compuestos en franca expansión, con productos en su mayoría de importación, como la amplia disponibilidad de materiales provenientes de recursos renovables a muy bajo costo, contribuyen favorablemente al desarrollo de un producto como el que se propone en este trabajo. En resumen, la propuesta es utilizar proteína de soja, quitosano, un agente entrecruzante y aserrín de madera (A), en diferentes proporciones, para obtener un biocomposito constituido por una matriz de uno o más bioplásticos entrecruzados y fibras de material celulósico conformado por termocompresión. En una fase final, en base a los resultados experimentales obtenidos con los prototipos de WBPCs desarrollados, se propone llevar a cabo el modelado empleando MEF (Método de los Elementos Finitos) para así simular y predecir el comportamiento de estos materiales cuando son sometidos a diferentes esfuerzos y sus posibles aplicaciones. La producción de estos WPCs conduciría a un aumento importante en el valor agregado de las materias primas, que son residuos o subproductos de muy bajo valor de las industrias aceitera, pesquera y maderera, y solucionaría, al menos en parte, los problemas ambientales y económicos generados por el excedente de estos residuos o subproductos.

\section{Actividades desarrolladas al presente}

Actividad 1: Obtención de aislado de proteína de soja.

Se realizó la extracción y desnaturalización de la proteína de la harina de soja siguiendo el procedimiento propuesto por L'hocine et al. (2006). La proteína se extrajo de la harina de soja con solución acuosa de $\mathrm{NaOH}$ a $60^{\circ} \mathrm{C}$ y, posteriormente, se agregó $\mathrm{HCl}$ (dis) hasta alcanzar el punto isoeléctrico de las proteínas de manera de lograr su precipitación. La separación de las mismas de la fase acuosa se llevó a cabo a temperaturas bajo cero.

\section{Actividad 2: Obtención de biocompositos con matriz de bioplástico y fibras de aserrín (WBPCs) por moldeo por termocompresión.}

Los WBPCs se prepararon utilizando una prensa hidráulica calefaccionada, manteniendo una presión de 70 bar a $100^{\circ} \mathrm{C}$ y $120^{\circ} \mathrm{C}$, entre 30 y 60 minutos. En ambos casos, la temperatura final se alcanzó con una velocidad de calentamiento de $2,5^{\circ} \mathrm{C} / \mathrm{min}$. Las probetas se prepararon con distintas relaciones matriz/fibras (SPI/A) entre 20/80 y 65/35, y variando la relación de reactivo entrecruzante a proteína de soja (GTA/SPI) en el rango 0-1 g GTA/g de SPI.

\section{Actividad 3: Caracterización de los WBPCs.}

Las muestras obtenidas se caracterizaron mediante ensayos de flexión, dureza, y absorción e hinchamiento en agua según norma internacional ASTM D1037-99 y se compararon con especificaciones técnicas de productos comerciales como el MDF. El estudio de la compatibilidad y cohesión entre la matriz y las fibras se realizó mediante microscopía electrónica de barrido (SEM). Por último, se expusieron a condiciones medioambientales para evaluar su comportamiento frente a factores como humedad ambiente, lluvia, exposición a rayos UV y ataque biológico.

\section{Resultados actuales}

La temperatura resultó un parámetro fundamental para obtener WBPCs con un buen acabado superficial y propiedades físicas y mecánicas que cumplen con especificaciones de productos comerciales. El uso combinado de GTA y alta temperatura $\left(120^{\circ} \mathrm{C}\right)$ permite obtener WBPCs con mayor dureza y rigidez que en muestras sin entrecruzante y preparadas a menor temperatura de curado.

Los WBPCs con las mejores propiedades mecánicas (Tabla 1) se obtuvieron para GTA/SPI=0,375 con SPI/A=20/80 y 35/65. Si bien estos biocompositos presentaron un módulo de ruptura (MOR) un 15-20\% inferior al mínimo requerido para tableros MDF, cumplen con los requisitos de densidad, dureza $(\mathrm{H})$ y módulo de elasticidad (MOE). El mínimo requerido para el MOR se podría alcanzar variando la temperatura y el tiempo de prensado para controlar el grado de entrecruzamiento de las proteínas.

Para SPI/A= 20/80 y distintas relaciones GTA/SPI, se observó que tanto la dureza $(\mathrm{H})$ como el módulo de elasticidad (MOE), alcanzaron sus valores máximos para relaciones GTA/SPI= 0,1875-0,375. A partir de GTA/SPI $=0,5$, tanto H como MOE decrecieron de forma importante (resultados no mostrados). Una posibilidad para explicar la disminución de estas propiedades es que el exceso de entrecruzante, que no reacciona con los grupos amino de las proteínas, se comporta como un plastificante impidiendo la interacción entre las moléculas de proteínas. Otra posibilidad es que, debido la alta concentración de agente entrecruzante, sólo uno de los grupos carbonilo de las moléculas de GTA reaccione con los grupos amino de las proteínas saturando estos. De esta manera, 
se inhibiría la posibilidad de conectar las cadenas de proteína unas con otras a través del otro grupo carbonilo del GTA, generándose así cadenas ramificadas en lugar de una red entrecruzada. En base a estos resultados, se concluye que una alta carga de fibras y un porcentaje intermedio de entrecruzante contribuyen a alcanzar un grado de interacción matriz-fibra de refuerzo y de entrecruzamiento proteína-GTA óptimos, obteniéndose así los máximos valores de $\mathrm{H}$ y MOE en estos WBPCs.

Tabla 1. Propiedades físicas y mecánicas de los WBPCs (Wood Bio-Plastic Composites) preparados por termocompresión a 70 bar y $120^{\circ} \mathrm{C}$ durante $30 \mathrm{~min}$.

\begin{tabular}{|c|c|c|c|c|c|c|c|c|}
\hline SPI/A & \multicolumn{2}{|c|}{$20 / 80$} & \multicolumn{2}{|c|}{$35 / 65$} & \multicolumn{2}{|c|}{$50 / 50$} & \multicolumn{2}{|c|}{$65 / 35$} \\
\hline GTA/SPI & 0 & 0,375 & 0 & 0,375 & 0 & 0,375 & 0 & 0,375 \\
\hline $\begin{array}{c}\text { Densidad } \\
{\left[\mathrm{kg} / \mathrm{m}^{3}\right]}\end{array}$ & $890 \pm 6$ & $930 \pm 11$ & $860 \pm 16$ & $940 \pm 2$ & $870 \pm 23$ & $990 \pm 14$ & $940 \pm 23$ & $950 \pm 23$ \\
\hline $\mathrm{H}^{\mathrm{a}}[\mathrm{N}]$ & $3072 \pm 72$ & $5720 \pm 330$ & $2331 \pm 173$ & $3985 \pm 710$ & $1384 \pm 180$ & $2664 \pm 108$ & $1287 \pm 140$ & $1647 \pm 114$ \\
\hline $\begin{array}{c}\mathrm{MOE}^{\mathrm{b}} \\
{\left[\mathrm{N} / \mathbf{m m}^{2}\right]}\end{array}$ & $2059 \pm 187$ & $2552 \pm 162$ & $1865 \pm 139$ & $2473 \pm 170$ & $1588 \pm 129$ & $2315 \pm 123$ & $1653 \pm 150$ & $1640 \pm 76$ \\
\hline $\begin{array}{c}\text { MOR }^{\mathbf{c}} \\
{\left[\mathrm{N} / \mathrm{mm}^{2}\right]}\end{array}$ & $21,0 \pm 0,6$ & $18,3 \pm 1,0$ & $20,5 \pm 0,8$ & $19,3 \pm 0,9$ & $15,1 \pm 1,2$ & $14,1 \pm 1,2$ & $17,3 \pm 0,7$ & $13,9 \pm 0,5$ \\
\hline
\end{tabular}

aDureza Janka, bMódulo de elasticidad y cMódulo de ruptura ASTM D1037-99.

Se observó por SEM que en ausencia de entrecruzante, la matriz tiene una textura heterogénea, formada por agregados de proteína y huecos entre estos (Fig. 1.A). Al adicionar GTA se observó una formación más continua y con menor número de huecos en la matriz de bioplástico, probablemente como resultado del mayor entrecruzamiento entre las cadenas de proteína a través de las moléculas de GTA (Fig. 1.B). Esto está en acuerdo con la mayor dureza y la mayor rigidez observadas para GTA/P=0,375 respecto de la muestra sin GTA.

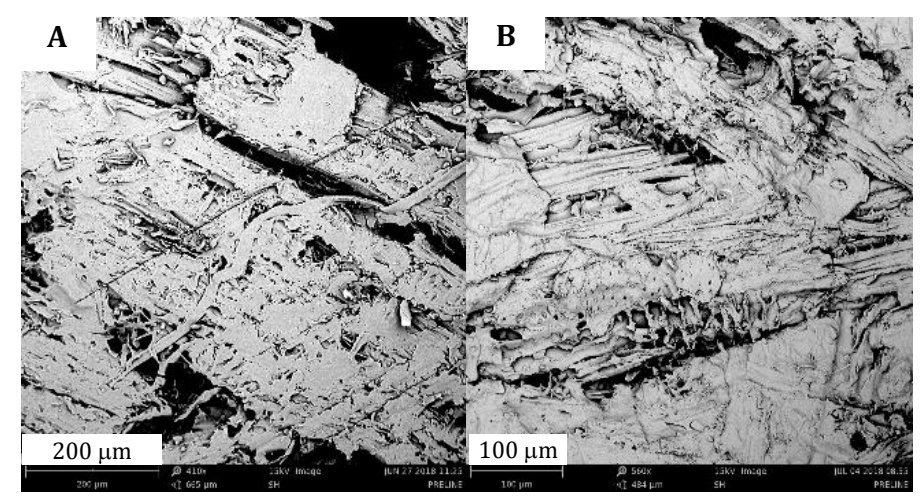

Figura 1. Imágenes SEM para relación SPI/A=20/80. A) GTA/SPI= 0. B) GTA/SPI=0,375.

El agregado de aserrín y de agente entrecruzante a la matriz bioplástica permite aumentar la rigidez, la dureza y el módulo de ruptura de estos WBPCs, en determinados rangos. Sin embargo, esto también produce un aumento en la absorción de agua e hinchamiento del WBPC. Estos resultados se pueden explicar a partir de mecanismos de interacción entre las cadenas de proteína, y de éstas con las fibras celulósicas y el agente entrecruzante (Wool and Sun, 2005). Por otra parte, se observó que aun cuando la absorción e hinchamiento por agua fue importante en los WBPCs, estos biocompositos prácticamente recuperaron su forma y espesor original luego del secado, siendo el hinchamiento residual menor al $8 \%$ en todas las muestras. Esto no ocurre con el MDF, el cual se deforma de manera irreversible durante la absorción de agua y posterior secado.

En cuanto a las probetas expuestas a las condiciones ambientales, luego de 5 meses las muestras con mayor cantidad de GTA no presentaron rasgos visibles de daño biológico (Fig. 2). Esto se puede atribuir al posible efecto antimicrobiano del GTA sin entrecruzar, ya que este dialdehído se utiliza como desinfectante y esterilizante de instrumentos, equipamiento y superficies en hospitales y quirófanos (Martí Solé et al, 1998). En cambio, las probetas con alto contenido de matriz y baja relación GTA/SPI fueron más rápida y fuertemente atacadas por hongos, sufriendo una marcada pérdida de peso debido a la desintegración de las mismas. 


\begin{tabular}{cccc}
\hline $\begin{array}{c}\text { Composición } \\
\text { SPI/A - GTA/SPI }\end{array}$ & Tiempo cero & Mes 2 & Mes 5 \\
\hline $\mathbf{2 0 / 8 0 - 0 , 5 0}$ & & & \\
\hline & & & \\
\hline $\mathbf{2 0 / 8 0 - 0 , 1 8 7 5}$ & & & \\
& & & \\
\hline $\mathbf{5 0 / 5 0 - 0 , 1 8 7 5}$ & & & \\
\hline
\end{tabular}

Figura2. Acción del medio ambiente sobre muestras de WBPC.

\section{Actividades futuras}

* Determinación de las condiciones óptimas para la disolución del quitosano. Como punto de partida, las soluciones de quitosano se prepararán con ácido acético.

* Preparación de biocompositos con matriz mixta de SPI y quitosano y, posterior caracterización en sus propiedades mecánicas y físicas. Se evaluará la posibilidad del empleo de otros agentes entrecruzantes como, por ejemplo, glioxal, y su influencia sobre las propiedades mecánicas y físicas de estos WBPCs

* Agregado de aditivos al biocomposito de bioplásticos y aserrín de madera. Algunos de los aditivos a considerar en esta parte son: agentes ignífugos, protectores UV, antimicrobianos y pigmentos.

* Aplicación de herramientas de simulación numérica mediante MEF para predecir el comportamiento en flexión de los WBPC.

\section{Referencias}

American Cancer Society (2014). Formaldehyde. Enlace: https://www.cancer.org/cancer/cancercauses/formaldehyde.html

ASTM Standard D1037 (1999). Standard test methods for evaluating properties of wood-base fiber and particle panel materials. ASTM International,West Conshohocken.

Bai, H. and Liu, X. (2017). Soy protein isolate-based films: preparation, properties, and applications. En: Soy-based Bioplastics, Smithers Rapra Technology, Shrewsbury. ISBN: 978-1-91024-224-7 (eBook).

Bogoeva-Gaceva, G., Avella, M., Malinconico, M., Buzarovska, A.,Grozdanov, A., Gentile, G. y Errico, M.E. (2007). Natural fiber eco-composites, Polym. Compos., 28, 98-107. doi: 10.1002/pc.20270.

Chan, C.M., Vandi, L-J., Pratt, S., Halley, P., Richardson, D., Werker, A. y Laycock, B. (2017). Composites of wood and biodegradable thermoplastics: a review, Polymer Rev., 58(3), 444-494.doi: 10.1080/15583724.2017.1380039.

L'hocine, L., Boye, J. I. y Arcand, Y. (2006). Composition and functional properties of soy protein isolates prepared using alternative defatting and extraction procedures, J. Food. Sci., 71(3), 137-145. doi: 10.1111/j.13652621.2006.tb15609.x. 
Li, B., Shan, C-L., Zhou, Q., Fang, Y., Wang, Y-l. ,Xu, F., Han, L-R., Ibrahim, M., Guo, L-B., Xie, G-L. y Sun, G-C. (2013). Synthesis, characterization, and antibacterial activity of cross-linked chitosan-glutaraldehyde, Mar. Drugs, 11, 15341552. doi: $10.3390 / \mathrm{md} 11051534$.

Kildeeva, N. R., Perminov, P. A., Vladimirov, L. V., Novikov, V. V. y Mikhailov, S. N. (2009). About mechanism of chitosan cross-linking with glutaraldehyde, Russ. J. Bioorg. Chem., 35, 360-369. doi: 10.1134/S106816200903011X

Martí Solé, M. C., Alonso Espadalé, R. M. y Constans Aubert, A. (1998) Desinfectantes: características y usos más corrientes, Instituto Nacional de Seguridad e Higiene en el Trabajo. España.

Mitra, B.C. (2014). Environment friendly composite materials: biocomposites and green composites, Def. Sci. J., 64(3), 244-261.doi: 10.14429/dsj.64.7323.

Netravali, A. N. y Chabba, S. (2003). Composites get greener, Mater. Today, 6(4), 22-29. ISSN:1369702.

Park S. R., Bae, D. H. y Rhee, K. C. (2000). Soy protein biopolymers cross-linked with glutaraldehyde, J. Amer. Oil Chem. Soc., 77(8), 879-884.doi: 10.1007/s11746-000-0140-3.

Rahman, K. S., Islam, N., Rahman, M., Hannan, O., Dungani, R. y Khalil, A. (2013). Flat-pressed wood plastic composites from sawdust and recycled polyethyleneterephthalate (PET): physical and mechanical properties, SpringerPlus2, 629-636. doi: 10.1186/2193-1801-2-629.

Schwarzkopf, M. J., Burnard, M. D. (2016). Wood-plastic composites - Performance and environmental impacts, En: Environmental impacts of traditional and innovative forest-based bioproducts, Springer, Singapore. doi: 10.1007/978-981-10-0655-5_2.

Selke, S. E. y Wichman, I. (2004). Wood fiber/polyolefin composites, Composites Part A, 35, 321-326.doi: 10.1016/j.compositesa.2003.09.010

Song, F., Tang, D., Wang, X. y Wang, Y. (2011). Biodegradable soy protein isolate-based materials: a review, Biomacromolecules,12, 3369-3380.doi: 10.1021/bm200904x.

Stark, N. M., Cai, Z. y Carll, C. (2010). Wood-based composite materials panel products, glued-laminated timber, structural composite lumber, and wood-nonwood composite materials, En: Wood handbook: wood as an engineering material, U.S. Department of Agriculture, Forest Service, Forest Products Laboratory, Madison. doi: 10.2737/FPL-GTR-190.

Wang, Y., Mo, X., Sun, X. y Wang, D. (2007). Soy protein adhesion enhanced by glutaraldehyde crosslink, J. Appl. Polym. Sci., 104, 130-136.doi: 10.1002/app.24675.

Wong S. S. y Jameson, D. M. (2012). Chemistry of protein and nucleic acid cross-linking and conjugation. Taylor \& Francis Group, Boca Raton. ISBN: 9781420007640 (eBook).

Wool, R.P y Sun, X. (2005). Bio-based polymers and composites. Elsevier Academic Press, Burlington. ISBN: 9780080454344(eBook). 